\title{
A QUEUEING SYSTEM WITH A FIXED ACCUMULATION LEVEL, RANDOM SERVER CAPACITY AND CAPACITY DEPENDENT SERVICE TIME
}

\author{
JEWGENI H. DSHALALOW \\ Department of Applied Mathematics, Florida Institute of Technology \\ Melbourne, FL 32901, USA \\ LOTFI TADJ \\ Department of Operations Research, Florida Institute of Technology \\ Melbourne, FL 32901, USA \\ (Received June 27, 1991)
}

\begin{abstract}
This paper introduces a bulk queueing system with a single server processing groups of customers of a variable size. If upon completion of service the queueing level is at least $r$ the server takes a batch of size $r$ and processes it a random time arbitrarily distributed. If the queueing level is less than $r$ the server idles until the queue accumulates $r$ customers in total. Then the server capacity is generated by a random number equals the batch size taken for service which lasts an arbitrarily distributed time dependent on the batch size.

The objective of the paper is the stationary distribution of queueing process which is studied via semi-regenerative techniques. An ergodicity criterion for the process is established and an explicit formula for the generating function of the distribution is obtained.
\end{abstract}

KEY WORDS AND PHRASES. Single-Server Queue, Queueing Process, Bulk Dependent Service, Accumulation, Markov Process, Markov Renewal Process, Semi-Regenerative Process.

1991 AMS SUBJECT CLASSIFICATION CODES. Primary 60K10, 60K25, Secondary 90B22, 90B25.

\section{INTRODUCTION.}

In many queueing systems with bulk service a server does not start service unless the number of waiting customers is at a certain fixed level. In this case the server is waiting for more arriving customers until the desired level is reached. A typical situation arises in computer network service, where every job to be done must go through a chain of computers (or parallel processors). The job can not get started until all necessary computer components are free. So the job (which now plays the role of a server) waits until the queue of waiting computers (in this case customers) accumulates a necessary group to run the job. A version of such a queue was modeled in Dshalalow and Russel [4]. A relevant modification of this model occurs when during waiting time a task can be reset up or being on a preliminary service insofar requiring a different (generally smaller) number of computer components by the time a group of the initially desired size becomes available. Such situations are common whenever a server, resting due to a queue accumulating more customers, lends a part of its capacity which perhaps may not be restored by the time the queue has reached the desired level. So by then the server begins to pro- 
cess a group of customers in accordance with the available capacity.

In the present paper the authors introduce and study a queueing model with an orderly Poisson input flow of customers and a single server of a variable capacity. The server usually takes a group of fixed size $r$ if such a group is available and processes it a random time with a given general distribution. Otherwise, the server idles until the level of the queue reaches level $r$. By then however capacity is a random number less than or equal to $r$ and it takes the corresponding batch for service which lasts a random time with a general distribution dependent on the batch size. The authors target the queueing process $\{Q(t)\}$ with continuous time parameter. They establish a steady state condition and obtain the stationary distribution for the process by using tools for semi-regenerative processes. An imbedded Markov chain is also given a detailed treatment.

\section{DESCRIPTION OF THE MODEL.}

We consider a servicing system with an infinite waiting room and a single channel processing a stream of customers described by an orderly stationary Poisson point process $\left\{\tau_{k} ; k \in \mathbb{N}\right\}$ with intensity $\lambda$. Denote $N(\cdot)$ the associated counting measure. Let $Q(t)$ denote the total number of customers in the system at time $t \geq 0$ and let $t_{0}=0, t_{1}, t_{2}, \ldots$ be the sequence of the successive completions of service of groups of customers. Defining $Q(t)$ as a right continuous process we introduce the imbedded process $Q_{n}=Q\left(t_{n}\right), n=1,2, \ldots$. Let $\sigma_{n}$ denote the service time of $n$th group of customers. If $Q_{n} \geq r$ then the server takes a group of size $r$ for service and immediately begins processing this group completing the service by $t_{n+1}$. In this case $\sigma_{n+1}=t_{n+1}-t_{n}$ and it is distributed according to a probability distribution function $B(B(0)=0)$ with a finite mean $b$. If $Q_{n}<r$ then the server waits $r-Q_{n}$ exponentially distributed phases, i.e. until $r-Q_{n}$ more customers arrive at the system reaching exactly level $r$ and only then the server is ready to begin service. But its capacity now becomes a random number $\gamma_{n+1}: \Omega \rightarrow\{1,2, \ldots, r\}$ generated by the begin of $n+1$ st service. We assume that $\gamma_{0}, \gamma_{1}, \gamma_{2}, \ldots$ are independent identically distributed random variables with the common probability mass function $\left(g_{1}, g_{2}, \ldots\right.$, $\left.g_{r}\right)$. Now given the server capacity $\gamma_{n+1}$ a group of the same size will be processed during a random time distributed according to $B_{\gamma_{n}} \in\left\{B_{1}, B_{2}, \ldots, B_{r}\right\}$, where the latter is a tuple of arbitrary probability distribution functions with finite means $\left\{b_{1}, b_{2}, \ldots, b_{r}\right\}$. In this case $t_{n+1}-t_{n}$ is the sum of server waiting time and the actual service time $\sigma_{n+1}$.

With the above formalism, the terms of the sequence $\left\{Q_{n}\right\}$ therefore satisfy the following recursive relation

$$
Q_{n+1}=\left\{\begin{array}{cc}
Q_{n}+\left(r-Q_{n}\right)-\gamma_{n+1}+V_{n+1}, & Q_{n}<r \\
Q_{n}-r+V_{n+1}, & Q_{n} \geq r
\end{array}\right.
$$

where $V_{n}=N\left(\sigma_{n}\right)$.

\section{ANALYSIS OF THE IMBEDDED PROCESS.}

From relation (2.1) and our assumption about the input stream it is obvious that $\{\Omega, \mathcal{F}$, $\left.\left(P^{x}\right)_{x \in E}, Q_{n} ; n=0,1, \ldots\right\} \rightarrow E=\{0,1, \ldots\}$ is a homogeneous Markov chain. Its transition probability matrix $A=\left(a_{i, j} ; i, j \in E\right)$ consists of two block matrices: The upper rectangular block with all positive elements

$$
a_{\imath},=\sum_{k=1}^{r} q_{k}, g_{k}, \quad i=0,1, \ldots, r-1, j \in E,
$$


where

$$
\begin{array}{ll}
q_{k j}=\int_{0}^{\infty} e^{-\lambda u} \frac{(\lambda u)^{j-r+k}}{(j-r+k) !} B(d u), & j \geq r-k \\
q_{k,}=0 & j=0, \ldots, r-k-1,
\end{array}
$$

and the lower block matrix which is an upper triangular matrix (with all positive elements on the main diagonal and above the main diagonal and zero elements below the main diagonal.) Thus $A$ is a $\Delta_{r, r}$-matrix, a special case of a class of $\Delta_{m, N}$-matrices studied by Abolnikov and Dukhovny [2]. According to the Abolnikov/Dukhovny criterion, the equilibrium of the process $\left(Q_{n}\right)$ is basically up to a certain quality of the generating function of the $r$ th row of matrix $A$. Let $A_{1}(z)$ denote the gencrating function of the $i$ th row of $A$.

PROPOSITION 1.

$$
\begin{array}{ll}
A(z):=A_{i}(z)=\sum_{k=1}^{r} z^{r-k} \beta_{k}(\lambda-\lambda z) g_{k}, & i=0, \ldots, r-1 \\
A_{1}(z)=z^{1-r} \beta(\lambda-\lambda z) & i \geq r
\end{array}
$$

where $\beta(\theta)$ and $\beta_{k}(\theta)$ are the Laplace-Stieltjes transforms of the corresponding probability distribution functions $B(x)$ and $B_{k}(x), k=1, \ldots, r$.

PROOF: Equations (3.1) and (3.2) are due to the relation (2.1) and straightforward probability arguments.

Let $\rho=\lambda b$. Then the following main result of this section holds true.

THEOREM 2. The imbedded Markov chain $\left(Q_{n}\right)$ is irreducible and aperiodic. It is recurrentpositive if and only if $\rho<r$. Under this condition, the generating function $P(z)$ of the steady state probability vector $P=\left(p_{0}, p_{1}, \ldots\right)$ of $\left(Q_{n}\right)$ satisfies the following formula:

$$
P(z)=\frac{\sum_{i=0}^{r-1} p_{i}\left[z^{r} A(z)-z^{i} \beta(\lambda-\lambda z)\right]}{z^{r}-\beta(\lambda-\lambda z)}
$$

where $A(z)$ is defined in (3.1).

PROOF: The chain is obviously irreducible and aperiodic. Due to the Abolnikov/Dukhovny criterion [2] applied to matrix $A$, the chain is recurrent-positive if and only if $A_{r}^{\prime}(1)=\rho<r$ and $A_{i}^{\prime}(1)<\infty, i=0, \ldots, r-1$. The latter is true due to $A_{i}^{\prime}(1)=\epsilon+\lambda \bar{g}$, where

$$
\begin{gathered}
\epsilon=r-E\left[\gamma_{1}\right] \\
\bar{g}=\sum_{k=1}^{r} b_{k} g_{k} .
\end{gathered}
$$

Formula (3.3) follows from the relation $P(\mathrm{z})=\sum_{\mathrm{i} \epsilon E} A_{i}(z) p_{i}$ and proposition 1 .

The determination of the unknown probabilities $p_{0}, \ldots, p_{r-1}$ is subject to the following

THEOREM 3. The unknown probabilities is a unique solution $p_{0}, \ldots, p_{r-1}$ of the following system of linear equations:

$$
\begin{gathered}
\sum_{i=0}^{r-1} p_{i} \frac{d^{k}}{d z^{k}}\left[A(z)-z^{i}\right] /=0, k=0, \ldots, k_{s}-1, s=1, \ldots, S, \\
\sum_{i=0}^{r-1} p_{i}\{\epsilon+r-i+\lambda \bar{g}-\rho\}=r-\rho,
\end{gathered}
$$

where $z$, are the roots of the function $z^{r}-\beta(\lambda-\lambda z)$ that belong to the closed unit ball $\bar{B}(0,1)$ in $C$ with their multiplicity $k_{\mathrm{s}}$ such that $\sum_{s=1}^{S} k_{s}=r-1$.

The proof of theorem 3 is similar to one in Abolnikov et al. [1].

DEFINITIONS AND NOTATIONS.
(i) Let $\beta=\left(\beta_{x} ; x \in E\right)^{T}$, where $\beta_{x}=E^{*}\left[t_{1}\right]=\left\{\begin{array}{cc}\frac{r-x}{\lambda}+\bar{g} & x<r \\ b & x \geq r\end{array}\right.$

(ii) Let $C$ denote the (stationary) capacity of the system, defined $C=\lambda P \beta$ (equals the ratio of 
the mean "service cycle" $P \beta$ and the mean interarrival time $1 / \lambda)$. Observe that the notion of the capacity of a system gocs back to the classical model $\mathrm{M} / \mathrm{G} / 1$, where $C$ is reduced to $C=\lambda b$ $=\rho$.

(iii) Let $c$ denote the (stationary) capacity of the server. Then obviously

$$
c=r \sum_{i=1}^{\infty} p_{i}+E\left[\gamma_{1}\right] \sum_{i=0}^{r-1} p_{i} .
$$

One remarkable property of the system in the equilibrium is that the capacities of the server and the system coincide.

PROPOSITION 4. Given the equilibrium condition $\rho<r$, the capacity of the system $C$ and the capacity of the server $c$ are equal.

PROOF: The equation $C=c$ follows from theorem 3, equation (3.7) after some algebra. Observe that equation (3.8) for $c$ can be rewritten in the form $C=c=r-\epsilon \sum_{i=0}^{r-1} p_{i}$.

EXAMPLE 5.

For $r=2$ and $B$ as exponential probability distribution function with parameter $1 / b$, there is obviously only one root of the denominator

$$
z^{2}-\beta(\lambda-\lambda z)=z^{2}-\frac{1}{1+\rho(1-z)}
$$

that belongs to the open unit ball $B(0,1)$ and it equals $z_{1}=\frac{1-\sqrt{1+4 \rho}}{2 \rho}$.

Equations (3.6) and (3.7) in theorem 3.3 are reduced to the simple system

$$
\left\{\begin{array}{l}
p_{0}(\epsilon+2+\lambda(\bar{g}-b))+p_{1}(\epsilon+1+\lambda(\bar{g}-b))=2-\rho \\
p_{0}\left(z_{1}^{2} A\left(z_{1}\right)-\frac{1}{1+\rho\left(1-z_{1}\right)}\right)+p_{1}\left(z_{1}^{2} A\left(z_{1}\right)-z_{1} \frac{1}{1+\rho\left(1-z_{1}\right)}\right)=0
\end{array}\right.
$$

having a unique solution $p_{0}$ and $p_{1}$.

\section{QUEUEING PROCESS WITH CONTINUOUS TIME PARAMETER.}

We first introduce a few notions.

DEFINITION 6. Let $T$ be a stopping time for a stochastic process $\left\{\Omega, \mathcal{F},\left(P^{x}\right)_{x \in E}, Z(t)\right.$; $t \geq 0\} \rightarrow(E, \mathfrak{B}(E)) .(Z(t))$ is said to have the locally strong Markov property at $T$ if for each bounded random variable $\zeta: \Omega \rightarrow E^{r}$ and for each Baire function $f: E^{r} \rightarrow \boldsymbol{R}, r=1,2, \ldots$, the following holds true

$$
E^{x}\left[f \circ \zeta \circ \theta_{T} \mid F_{T}\right]=E^{Z_{T}}[f \circ \zeta] P^{x} \text {-a.s. on }\{T<\infty\},
$$

where $\theta_{y}$ is the shift operator.

DEFINITION 7. A stochastic process $\left\{\Omega, \mathcal{F},\left(P^{x}\right)_{x \in E}, Z(t) ; t \geq 0\right\} \rightarrow(E, \mathbb{B}(E))$ with $E \preceq N$ is called semi-regenerative if

a) there is a point process $\left\{t_{n}\right\}$ on $\mathbf{R}_{+}$such that $t_{n} \rightarrow \infty(n \rightarrow \infty)$ and that each $t_{n}$ is a stopping time relative to the canonic filtering $\sigma\left(Z_{y} ; y \leq t\right)$,

b) the process $(Z(t))$ has the locally strong Markov property at $t_{n}, n=1,2, \ldots$,

c) $\left\{Z\left(t_{n}+0\right), t_{n} ; n=0,1, \ldots\right\}$ is a Markov renewal process.

It is obvious that the process $\{Q(t)\}$ has the locally strong Markov property at the stopping time $t_{n}, n=1,2, \ldots$, and thus $\{Q(t)\}$ is a semi-regenerative process with respect to the sequence $\left\{t_{n}\right\}$ (see definitions 6 and 7.)

DEFINITION 8. Let $\left(X_{n}, t_{n}\right)$ be an irreducible aperiodic Markov renewal process with a discrete state space $E$. Denote $\beta_{x}=E^{x}\left[t_{1}\right]$ the mean sojourn time of the Markov renewal process in state $\{x\}$ and let $\beta=\left(\beta_{x} ; x \in E\right)^{\mathrm{T}}$. Suppose that the imbedded Markov chain $\left(X_{n}\right)$ is ergodic and that $P$ is its stationary distribution. We call $P \beta$ the mean inter-renewal time. We call the Markov renewal process recurrent-positive if its mean inter-renewal time is finite. An irreducible 
aperiodic and recurrent-positive Markov renewal process is called ergodic.

DEFINITION 9. Let $\left\{\Omega, \mathcal{F},\left(P^{x}\right)_{x \in E}, Z(t) ; t \geq 0\right\} \rightarrow(E, \mathfrak{B}(E))$ be a semi-regenerative process relative to the sequence $\left\{t_{n}\right\}$ of stopping times. Introduce the probability

$$
K_{j k}(t)=P^{j}\left\{Z(t)=k, t_{1}>t\right\}, j, k \in E .
$$

We will call the functional matrix $K(t)=\left(K_{j k}(t) ; j, k \epsilon E\right)$ the semi-regenerative kernel.

Before stating the main result of this section, we will recall the main convergence theorem and its corallary.

THEOREM 10. (The Main Convergence Theorem, cf. Çinlar [3], p. 347). Let $\left\{\Omega, \mathcal{T},\left(P^{x}\right)_{x \in E}\right.$, $Z(t) ; t \geq 0\} \rightarrow(E, \mathfrak{B}(E))$ be a semi-regenerative stochastic process relative to the sequence $\left\{t_{n}\right\}$ of stopping time and let $K(t)$ be the corresponding semi-regenerative kernel. Suppose that the associated Markov renewal process is ergodic and that the semi-regenerative kernel is Riemann integrable over $\mathbf{R}_{+}$. Then the stationary distribution $\pi=\left(\pi_{x} ; x \in E\right)$ of the process $(Z(t))$ exists and it is determined from the formula:

$$
\pi_{k}=\frac{1}{P \beta} \quad \sum_{j \epsilon E} p, \int_{0}^{\infty} K_{j k}(t) d t, k \in E .
$$

COROLLARY 11. Denote $H=\left(h_{j k} ; j, k \in \mathrm{E}\right)=\int_{0}^{\infty} K(t) d t$ the integrated semi-regenerative kcrnel, $h_{j}(z)$ the generating function of $j$ th row of matrix $H$ and $\pi(z)$ the generating function of vector $\pi$. Then the following formula holds true

$$
\pi(z)=\frac{1}{P \beta} \sum_{j \in E} p, h_{j}(z) .
$$

PROOF. From (4.1) we get an equivalent formula in matrix form $\pi=\frac{P H}{P \beta}$. Finally, formula (4.2) is the result of elementary algebraic transformations.

The following is the main result of this section.

THEOREM 12.

(i) The limiting distribution $\pi=\left(\pi_{0}, \pi_{1}, \ldots\right)$ of the process $\{Q(t)\}$ exists if and only if $\rho<r$.

(ii) The generating function $\pi(z)$ of $\pi$ satisfies the following formula:

$$
\dot{\pi}(z)=\sum_{k \geq 0} \pi_{k} z^{k}=\frac{1}{C} \frac{1-z^{r}}{1-z} P(z)-\frac{1}{C} z^{r} \alpha(z) \sum_{i=0}^{r-1} p_{i},
$$

where $\alpha(z)=\left\{\begin{array}{cc}\sum_{s=0}^{r-1} g_{s} \beta_{s}(\lambda-\lambda z) \frac{1-z^{r-s}}{1-z}, & r \geq 2 \\ 0, & r=1\end{array}\right.$

PROOF: The validity of assertion $(i)$ is subject to a routine proof.

(ii) Let $K(t)=\left(K_{j k}(t) ; j, k \in E\right)$ denote the semi-regenerative kernel (definitions 8 and 9 ), where $K_{j k}(t)=P^{i}\left\{Q(t)=k, t_{1}>t\right\}$. By elementary probability arguments we deduce that

$$
\begin{aligned}
& K_{j k}(t)=e^{-\lambda t \frac{(\lambda t)^{k-j}}{(k-j) !}} \quad 0 \leq j \leq k<r \\
& K_{j k}(t)=\int_{0}^{t} \lambda e^{-\lambda(t-u)} \frac{[\lambda(t-u)]^{r-j-1}}{(r-j-1) !} e^{-\lambda u} \frac{(\lambda u)^{k-r}}{(k-r) !} \sum_{s=1}^{r}\left[1-B_{s}(u)\right] g_{s} d u, \\
& K_{j k}(t)=e^{-\lambda t} \frac{(\lambda t)^{k-j}}{(k-j) !}[1-B(t)], \quad r \leq j \leq k, \\
& K_{j k}(t)=0, \quad 0 \leq k<j, j>0
\end{aligned}
$$

$0 \leq j \leq r-1, k \geq r$

Clearly, $K(t)$ is Riemann integrable over $\mathbf{R}_{+}$and denote

$$
H=\int_{0}^{\infty} K(t) d t
$$

Apply the main convergence theorem for semi-regenerative processes in the form of

$$
\pi=\frac{P H}{P \beta}
$$

(see theorem 10). Here $P \beta$ can be expressed through the capacity of the system $C=\lambda P \beta$ which 
is $C=r-\epsilon \sum_{i=1}^{r-1} p_{\imath}$ due to proposition 4.

(4.9) can be expressed in terms of the generating functions, as

$$
\pi(z)=\frac{\lambda}{C} \quad \sum_{j \in \mathrm{E}} h(z) p_{j}
$$

where $h_{j}(z)$ is the generating function of the $j$ th row of the matrix $H$ (theorem 11.) Now formula (4.3) follows from (4.10) and from the expressions for $h_{j}(z)$ :

$$
\begin{array}{cc}
h_{j}(z) \lambda(1-z)=z^{j}-z^{r} \sum_{:=1}^{r} g_{s} \beta_{0}(\lambda-\lambda z), & j=0, \ldots, r-1 \\
h_{j}(z) \lambda(1-z)=z^{j}(1-\beta(\lambda-\lambda z)), & j \geq r .
\end{array}
$$

EXAMPLES 13.

1) For $r=2, \quad \pi(z)=\frac{1}{C}(1+z) P(z)-\frac{1}{C} z^{2} g_{1} \beta_{1}(\lambda-\lambda z)\left(p_{0}+p_{1}\right)$,

where $C=2-g_{1}\left(p_{0}+p_{1}\right)$. If in addition $B$ is an exponential distribution then $p_{0}$ and $p_{1}$ satisfy system (3.9) in example 5 .

2) The expected length of an idle period of the server in the equilibrium satisfies the below formula (due to straightforward probability arguments):

$$
\bar{I}=\frac{\sum_{i=0}^{r-1} p_{i} \frac{r-i}{\lambda}}{\sum_{i=0}^{r-1} p_{i}} .
$$

The probability that the server is idle in the stationary mode is

$$
\sum_{i=0}^{r-1} \pi_{i}=\frac{\bar{I}}{\bar{I}+\bar{B}}
$$

where $\bar{B}$ denotes the mean busy period which thus can be expressed in terms of known values:

$$
\bar{B}=\frac{\sum_{i=r}^{\infty} \pi_{i}}{\sum_{i=0}^{r 1} \pi_{i}} \bar{I} .
$$

\section{REFERENCES}

1. ABOLNIKOV, L., DSHALALOW, J. and DUKHOVNY, A.M., On some queue length controlled stochastic processes, Journ. Appl. Math. Stoch. Analysis, 3, No. 4 (1990), 227-244.

2. ABOLNIKOV, L.M. and DUKHOVNY, A.M., Necessary and sufficient conditions for the ergodicity of Markov chains with transition $\Delta_{m, n}\left(\Delta_{m, n}^{\prime}\right)$ matrix, Journ. Appl. Math. Simul., 1 , No. 1 (1987), 13-24.

3. ÇINLAR, E., Introduction to Stochastic Processes, Prentice Hall, 1975.

4. DSHALALOW, J. and RUSSEL, G., On a single server queue with fixed accumulation level, state dependent service, and semi-Markov modulated input flow, (submitted to International Journal of Mathematics and Mathematical Sciences.) 


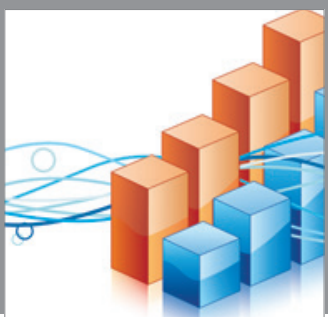

Advances in

Operations Research

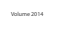

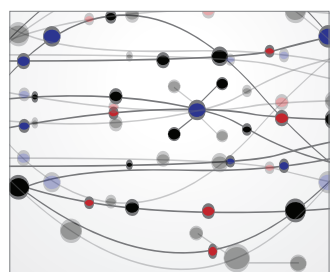

\section{The Scientific} World Journal
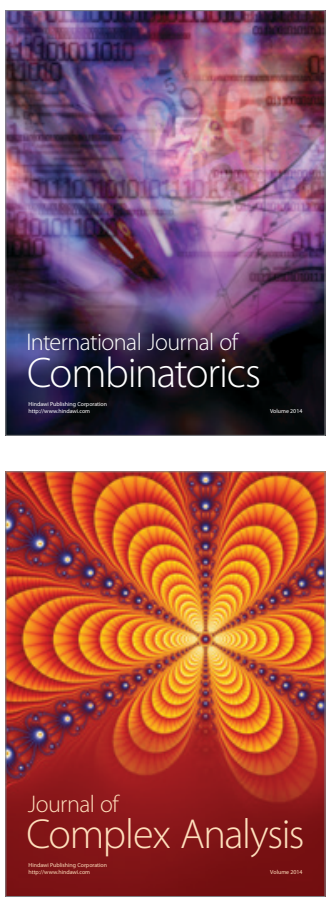

International Journal of

Mathematics and

Mathematical

Sciences
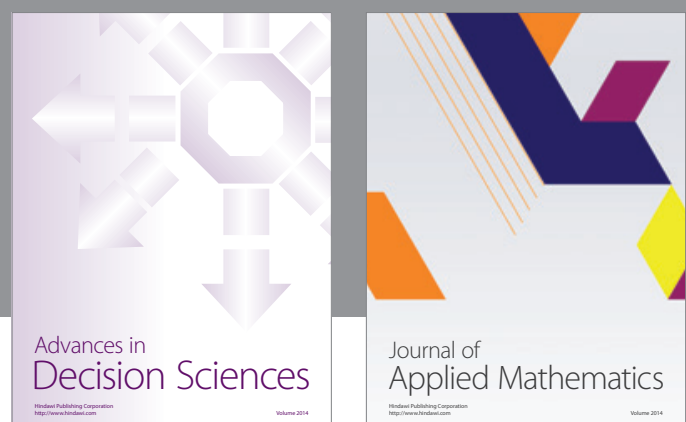

Journal of

Applied Mathematics
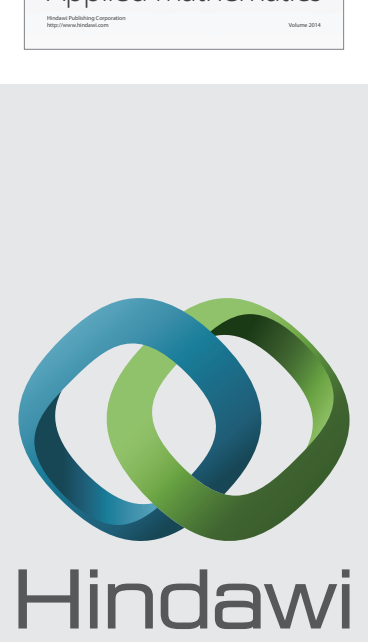

Submit your manuscripts at http://www.hindawi.com
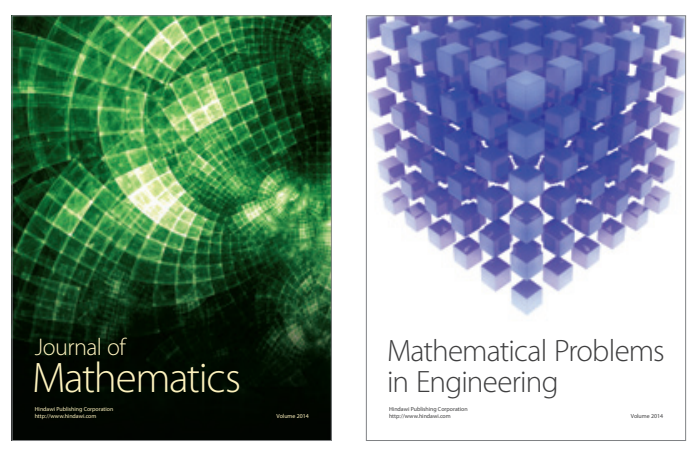

Mathematical Problems in Engineering
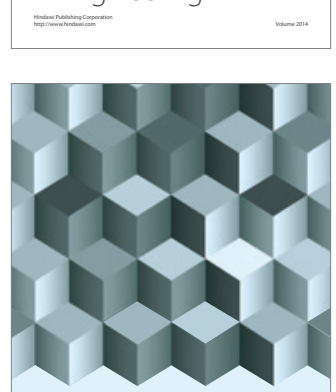

Journal of

Function Spaces
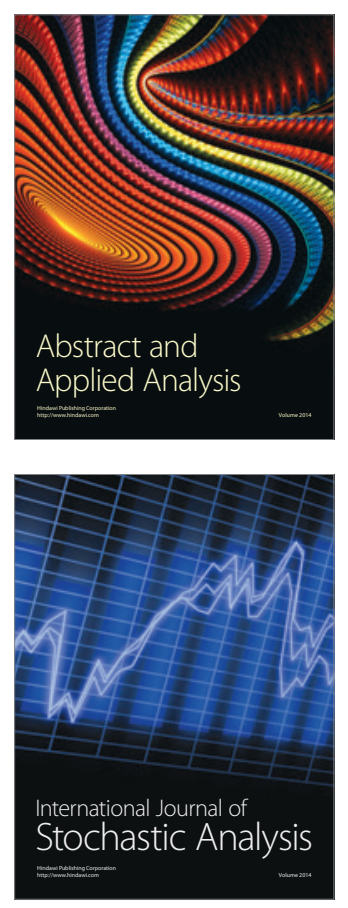

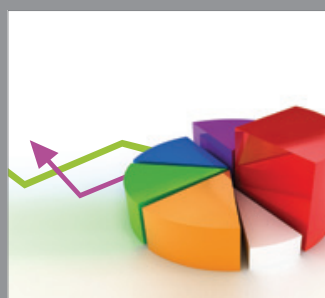

ournal of

Probability and Statistics

Promensencen
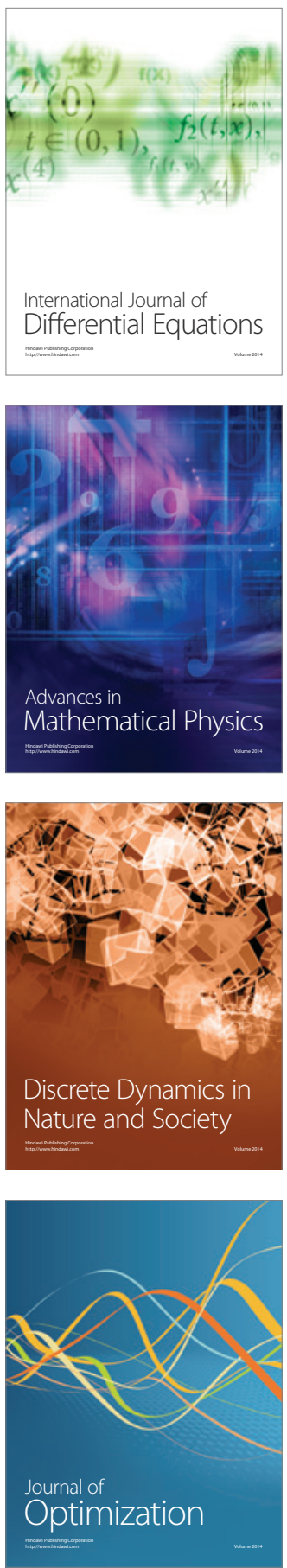